\title{
Cerebra Thevetia poisoning: A prospective study at a tertiary care hospital, Kerala
}

\author{
Radhika R. H.
}

Professor, Dept. of Forensic Medicine \& Toxicology, Gouri Devi Institute of Medical Sciences, Rajbandh, West Bengal, India

*Corresponding Author:

Email:rhr_doc@yahoo.com

\begin{abstract}
Yellow oleander or cerebra Thevetia poisoning is commonly encountered from southern parts of India unlike the northern parts. Because of its beautiful flowers, people plant yellow oleander tree in their garden \& compound as a hobby without knowing its poisonous action. This prospective study was carried out in the Department of Forensic Medicine \& Toxicology, Karuna Institute of Medical Sciences \& Hospital, Palakkad, Kerala for a period of 2 year from January 2015 to February 2017.25 cases which presented with yellow oleander consumption to emergency ward were enrolled in this study. A detailed history of patient demographic profile, number of seeds consumed, time of consumption, time of presentation to hospital, any form of treatment given before presenting to hospital, detailed clinical assessment of the patient and routine blood investigations were done and a 12 lead electrocardiogram was recorded. Patients were monitored for electrolyte imbalance every 6th hourly and a continuous cardiac monitoring was done to recognize cardiac arrhythmias and ECG was recorded at the admission and every 2nd hourly for 48 hours. Out of 25 cases studied, 9 cases (36\%) did not show any ECG abnormality. Remaining 16 cases $64 \%$ showed ECG abnormalities. Various ECG abnormalities noted amongst the patients were sinus bradycardia $(<40 / \mathrm{pm}) 8$ cases $(50 \%)$, ST wave inversion and T wave inversion 2 cases (12.75\%), AV dissociation 3 cases (18.75\%), ventricular tachycardia 2 cases (12.75\%), ventricular fibrillation 1 case $(6.25 \%)$, and all these cases were shifted to ICU immediately. Serum calcium and serum potassium levels were slightly elevated in all the patients who showed ECG abnormalities.
\end{abstract}

Keywords: Yellow oleander, Cardiac arrhythmias, Suicide, Cardiac glycosides.

\section{Introduction}

A poison is a substance which, when administered, inhaled or ingested, is capable of acting deleteriously on the human body. Thus, there are really no limits, between a medicine and a poison, for a medicine in a toxic dose is a poison and a poison in a small dose may be a medicine means, it depends on dose/quantity only. ${ }^{1}$ The incidence of poisoning in India is among the highest in the world, and it is estimated that more than 50,000 people die every year from toxic exposure. ${ }^{2}$ Common causes of poisoning in India include insecticides, household chemicals such as sanitation fluids, plants, animal bites, and stings. According to one study, plant poison accounted for $20 \%$ mortality rate of all poisonous exposures. ${ }^{3}$ Most exposures are accidental and occur in children and are mild. Life-threatening exposures occur in adults who intentionally ingested in some form or other. Poisonous plants encountered in India include ${ }^{4}$ (1) contact irritant poisons - castor, calotropis, croton, glory lily, marking nut, papaya, and red pepper; (2) cardiotoxic glycosides-aconite, autumn crocus, and common oleander; (3) neurotoxic plants-calotropis, cassava, datura, strychnos; (4) hepatotoxic plants-neem; and (5) miscellaneous toxic plants and plant products - areca nut and Cleistanthus collinus. There are no specific data available in India as of the most common plants and their toxicity pattern. Poisonous plants are those which cause serious problems or even death occur, if a small quantity of its stem, leaves, seeds, fruits and roots are ingested. They are sometimes referred as botanical weapons when used for committing crimes like theft, burglary, murder etc. In the present study, an effort was made to know the demographic profile and cardiac manifestations on consumption of cerebra Thevetia plant seeds. Oleander plant is grown widely as ornamental plant. Different varieties are known: pink oleander, yellow oleander (Thevitia peruviana), and white oleander. All parts of the plant including nectar are poisonous. Inhalational poisoning has been reported when dried twigs are burnt as biomass fuel. Consumption of seeds of Thevetia is the mode of exposure of poisoning in various age groups and the severity of poisoning depends upon the number of seeds/ leaves consumed. Some studies showed that consumption of more than 5 crushed seed was associated with significant manifestation, the fatal dose being 8 to 10 seeds, $15-20 \mathrm{~g}$ of root, 5 to 10 leaves. ${ }^{5}$ The toxic principles are cardiac glycosides similar to digoxin. They are thevetin, thevetoxin, nerifolin, peruvoside, ruvoside and cerberinoleandrin, oleandrigenin, and oleandroside. Most cases recover well with supportive therapy alone compared to fatal poisoning cases that develop various cardiac dysfunctions ranging from sinus bradycardia to heart block of various degree and ventricular fibrillation. Information on its poisoning and knowledge of its fatal effects to the people of the surrounding locality of study area was found to be limited and hence the present study was taken up to be carried out with the objective to study demographic profile and cardiovascular manifestations associated with Thevetia poisoning cases.

\section{Materials and Methods}

This descriptive study was carried out in the Department of Forensic Medicine \& Toxicology, 
Karuna Institute of Medical Sciences \& Hospital, Palakkad, Kerala for a period of 2 year from January 2015 to February 2017. Total of 25 cases of suspected cerebra Thevetia poisoning that were admitted to the emergency department of the hospital were included in the study. Cases of unknown poisoning and Organo Phosphorus poisoning were excluded from the study. This a record based study where the demographic profile of the patient, number of seeds consumed, time of consumption and their arrival to the hospital were entered in a pre formed proforma. A 12-lead standard electrocardiogram, blood investigations like serum calcium, magnesium, potassium, sodium taken for each patient were also included in the study. The treatment of the patients that included gastric lavage using activated charcoal and symptomatically for the initial gastro intestinal discomfort like vomiting, diarrhea, abdominal pain was studied. It is observed that those who showed certain cardiac abnormalities on ECG at the time of admission with symptoms like syncope attack, cyanosis, breathlessness, bradycardia, and hypotension were shifted immediately to ICU and monitored round the clock for 48 hours and electrolyte abnormalities were monitored every $4^{\text {th }}$ hourly along with the symptomatic care. Bradycardia related arrhythmias were managed with intravenous atropine and adrenalin, and lidocaine along with correction of fluid and electrolyte imbalance. The prognosis of the patient directly related to the total number of seeds consumed and high risk cases were referred to hospitals with better facilities for treatment; however fatality was not encountered in the present study.

\section{Results}

Incidence of cerebra Thevetia poisoning was common among males $72 \%$ cases than females $28 \%$ cases, majority were in the age group of $21-30$ years (44\%) cases, less common among 11-20 years (20\%) cases. Most of the patient had consumed the seeds of cerebra Thevetia during day between 6AM to 6PM 76\% cases compared to $24 \%$ cases consumed between $6 \mathrm{PM}$ to 6AM. Almost all cases $92 \%$ were brought to the hospital within 2 hours after the consumption of seeds, except for $8 \%$ cases brought between 2 to 4 hours. Out of 25 cases, 9 cases $36 \%$ had consumed 2 seeds which did not show any ECG abnormality. Remaining 16 cases $64 \%$ showed ECG abnormalities and also the total number of seeds consumed was more than 3 seeds, maximum seed consumed was 5 . Table 1 shows various ECG abnormalities amongst the patients were sinus bradycardia $(<40 / \mathrm{pm}) \quad 8$ cases $(50 \%)$, ST wave inversion and $\mathrm{T}$ wave inversion 2 cases $(12.75 \%), \mathrm{AV}$ dissociation 3 cases (18.75\%), ventricular tachycardia 2 cases $(12.75 \%)$, ventricular fibrillation 1 cases $(6.25 \%)$ and all these cases were shifted to ICU immediately. Serum calcium and serum potassium levels were slightly elevated in all the patients who showed ECG abnormalities. Other hematological and biochemical parameters were within normal limits in all the patients.

Table 1: Various ECG abnormalities seen in cerebra thevetia poisoning patients

\begin{tabular}{|l|c|c|}
\hline ECG abnormality & Total number $(\mathbf{n})=\mathbf{~ 1 6}$ & Percentage (\%) \\
\hline Sinus bradycardia & 8 & 50 \\
\hline ST wave, Twave inversion & 2 & 12.5 \\
\hline Atrioventricular Dissociation & 3 & 18.75 \\
\hline Ventricular Tachycardia & 2 & 12.5 \\
\hline Ventricular Fibrillation & 1 & 6.25 \\
\hline
\end{tabular}

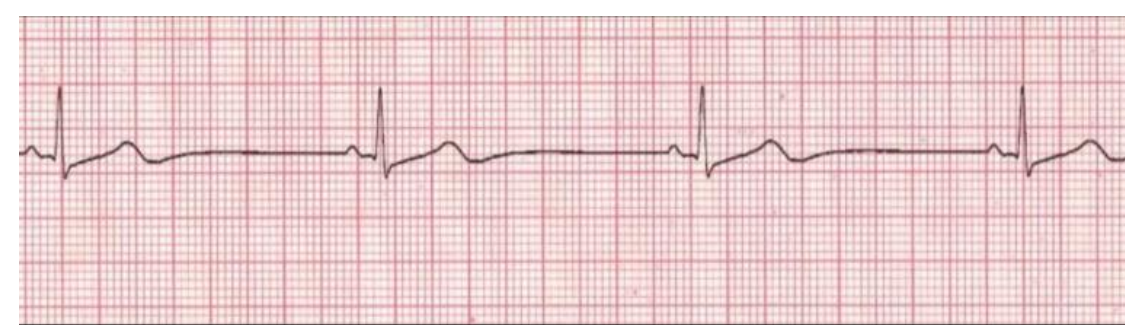

Fig. 1: Sinus bradycardia

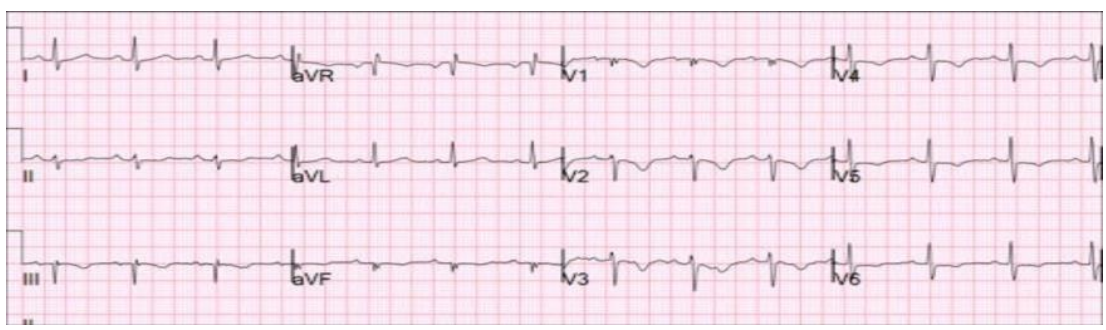

Fig. 2: ST inversion and T inversion 


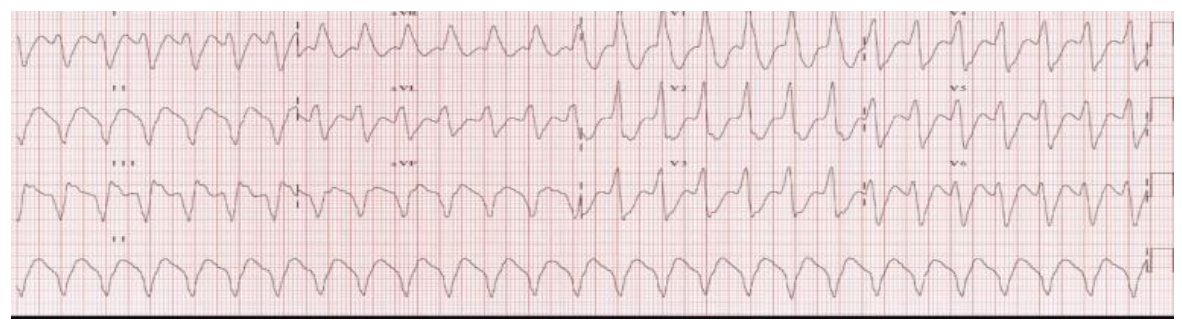

\section{Fig. 3: Ventricular tachycardia}

\section{Discussion}

The digitalis glycosides are by far the best known of the cardiac glycosides but many hundreds of others have been identified in different species of plants from at least 12 different families. The Apo-cyanaceae family of plants is source of African arrow poisons and also contains many of the most beautiful but deadly tropical flowers such as cerebra odollum, common oleander and yellow oleander. The yellow oleander is another notoriously toxic plant that contains at least eight different cardiac glycosides. ${ }^{6}$ All parts of the plant are dangerous, especially the seeds. The active principle of this plant are glycosides namely thevetin, thevetoxin $\&$ cerebrin. Both thevetin \& thevetoxin are cardiotoxic $\&$ their actions resemble that of digitalis. On the other hand, cerberin has an action like strychnine. These cardiac glycosides affect cardiovascular system (CVS), central nervous system (CNS) \& gastrointestinal system. Of these, effects on CVS are most important. The toxins after binding to a site on cell membrane, produce reversible inhibition of $\mathrm{Na}+\mathrm{K}+$ Adenosine Triphosphatase pump which causes increased intracellular $\mathrm{Na}+\&$ decreased intracellular $\mathrm{K}+$. This elevated intracellular $\mathrm{Na}+$ concentration allows more $\mathrm{Ca}++$ influx into the myocardial cells via a $\mathrm{Na}+-\mathrm{Ca}++$ exchanger. As a result of excessive $\mathrm{Ca}++$ within myocardium, widespread cardiac contraction occurs \& manifest clinically as premature ventricular contractions. ${ }^{7}$ Cardiac glycosides also have vagotonic effects resulting in bradycardia \& heart blocks. In addition, inhibition of $\mathrm{Na}+-\mathrm{K}+$ ATPase in skeletal muscle results in excess $\mathrm{K}+$ in extra cellular fluid with concomitant manifestations of hyperkalaemia. ${ }^{8}$

Ingestion of Oleander seeds or leaves is a common cause of accidental poisoning worldwide, particularly among children. The Oleander has been used for suicide, homicide, abortion and as herbal remedies in India, Thailand, Brazil and elsewhere.

Most of the patients in this study $72 \%$ were young males less than 30years of age who consumed the oleander seeds and some had accidentally consumed under the influence of consumption of alcohol and some for committing suicide due to work related stress, financial problems and other reasons given by the patients. Female patients were less $28 \%$ in this study. In a study at Colombo females were 59\%. ${ }^{9}$ Majority were of age 21 to 30 years, similar results were seen at other studies. ${ }^{10-12}$ Consumption of seeds either accidentally or for committing suicide occurred most commonly during day time and only $24 \%$ cases patients had consumed at night time. This perhaps could be due to work related stress and as the seeds were easily accessible for consumption not exactly knowing that a small dose of seeds can be fatal. However majority of the patients $92 \%$ arrived at the hospital within 2 hours after consumption and only 2 cases took 2 to 4 hours to reach the hospital for treatment as the study area being a tertiary care hospital surrounding the most of rural belt was within the reach of most of the patients who had consumed the seeds.

In the present study patients who had consumed two seeds presented with vomiting, diarrhea, headache, dizziness, dilated pupils, drowsiness. Along with these varying types of arrhythmia and features of shock were also predominant who had consumed more than three oleander seeds and maximum seeds were consumed were five in number however there was no fatality in this study. A normal ECG was found on presentation in $36 \%$ of patients. In the remaining $64 \%$, conduction abnormalities of the sinus node or AV node were detected as seen in table 1. The common electrocardiographic abnormalities reported are bradycardia, ST segment depression, inversion of $\mathrm{T}$ waves, AV dissociation, and in large doses ventricular tachycardia and ventricular fibrillation. On Biochemical analysis revealed hyperkalaemia and hyperkalemic acidosis, however serum sodium and other parameters were within normal limits in all the patients who showed ECG abnormalities. Similar predominant cardiac manifestations were also noted by other authors. ${ }^{6,10-12}$

All the patients were given gastric lavage with activated charcoal on admission and treated symptomatically. Those who presented with cardiac abnormalities on ECG were shifted immediately to Intensive care unit and treated with intravenous atropine and adrenalin, and lidocaine along with correction of fluid and electrolyte imbalance. Of 25 cases 11 cases were referred to higher centers at the earliest for further management and for treatment of cardiac abnormalities and remaining 14 cases were discharged from the hospital. Similar study showed patients were referred to higher centers. ${ }^{6}$ In one study it was observed that atropine was sufficient for reversing bradycardia, though effects of atropine on outcome have not been formally assessed and also it is stated that several cases of oleander poisoning were managed with 
supportive measures and atropine with good outcome. ${ }^{13}$ The outcome of yellow oleander poisoning depends on the number of seeds or the amount of the plant ingested and how quickly the management has started and complete recovery is likely if the patient survives the first 24 hours of ingestion. ${ }^{10-12}$ Majority of the patients were brought to hospital within two hour of ingestion and resuscitation was done promptly with the assistance of cardiology department and perhaps this saved patients from this deadly poison.

\section{Conclusion}

It is interesting that oleander poisoning can be fatal with relatively small amounts ingested. There is a poor correlation between the number of seeds ingested and the severity of cardio toxicity. Death has occurred after ingestion of one or two seeds; in contrast, patients have survived after consuming ten or more seeds without requiring specialized treatments. Suicide attempt due to consumption of oleander seeds seems to be under recognized problem in India and practicing physicians should understand the potential lethal properties of oleander and its availability throughout the world. Future studies will need to assess the relative importance of cardiac glycoside levels and electrolyte disturbances at presentation to the emergency department as determinants of mortality.

\section{References}

1. Narayan Reddy KS. Essentials of Forensic Medicine and Toxicology, 33rd Edn. Hyderabad, India Jaypee Brothers Medical Publication (P) Ltd, 2014.

2. Boesche, Roger (2003) "Kautilya's Arthasastra on War and Diplomacy in Ancient India". The Journal of Military History 67:9-37. ISSN 0899-3718.
3. Maharani B, Vijayakumari N. Profile of poisoning cases in a Tertiary care Hospital, Tamil Nadu, India. J Appl Pharm Sci. Jan-2013, Vol. 3(01), pp.091-094.

4. Pillay VV. Modern Medical Toxicology, 4th edn. New Delhi, India: Jaypee Brothers Medical Publication (P) Ltd, 2013.

5. Khajja et al: Forensic Study of Indian Toxicological Plants as Botanical Weapon (BW): A Review $J$ Environment Analytic Toxicol 2011, 1:4.

6. Jalal Z, Amir: Cardiac Findings in Acute Yellow Oleander Poisoning J Cardiovasc Dis Res. 2010 JanMar;1(1):27-28.

7. Mollah A H et al: Yellow oleander poisoning : a case report Journal of Nepal Medical Association, 2002:41:331-334.

8. Douglas R. L. Plant Poisoning, E-Medicine Journal, May, 2001, 2(5):1 (www.acep.org)

9. M Eddleston, et al Acute yellow oleander (Thevetia peruviana) poisoning: cardiac arrhythmias, electrolyte disturbances, and serum cardiac glycoside concentrations on presentation to hospital Heart 2000;83:301-306.

10. S. Lokesh, R. Arunkumar : A clinical study of 30 cases of Acute Yellow Oleander Poisoning Journal of Current Trends in Clinical Medicine \& Laboratory Biochemistry1, Vol. 1, Issue 2, July-September 2013.

11. Pathare A V, Patil R R, Chikhalikar A A, Dalvi S G : Rare poisoning with Cerebra thevetia (a case report). $J$ Postgrad Med 1987;33:216.

12. Khan I et al: Acute Cardiac Toxicity of Nerium Oleander/Indicum Poisoning (Kaner) Poisoning Heart Views: The Official Journal of the Gulf Heart Association. Oct-Dec 2010;11(3)115.

13. Mandal L: Yellow Oleander- Thevetia peruvianaPoisoning Postgraduate Medical Journal of NAMS Vol. 12. Number 2. Jul-Dec 2012. 